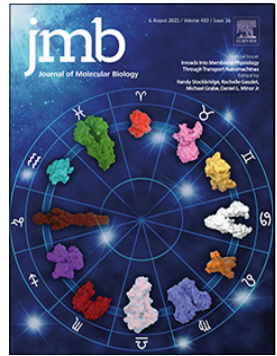

\title{
The Crystal Structure of the $\mathrm{Ca}^{2+}$-ATPase 1 from Listeria monocytogenes reveals a Pump Primed for Dephosphorylation
}

\author{
Sara Basse Hansen ${ }^{1,2}$, Mateusz Dyla ${ }^{1,2}$, Caroline Neumann ${ }^{1,2}$, \\ Esben Meldgaard Hoegh Quistgaard ${ }^{1,2}$, Jacob Lauwring Andersen ${ }^{1,2 \dagger}$, \\ Magnus Kjaergaard ${ }^{1,2,3,4}$ and Poul Nissen ${ }^{1,2,4 *}$
}

1 - Department of Molecular Biology and Genetics, Aarhus University, Denmark

2 - The Danish Research Institute for Translational Neuroscience (DANDRITE), Nordic EMBL Partnership for Molecular Medicine, Denmark

3 - Aarhus Institute of Advanced Studies (AIAS), Denmark

4 - The Danish National Research Foundation Center for Proteins in Memory (PROMEMO), Denmark

Correspondence to Poul Nissen: Department of Molecular Biology and Genetics, Aarhus University, Denmark. pn@mbg.au.dk (P. Nissen)

https://doi.org/10.1016/j.jmb.2021.167015

Edited by Daniel L. Minor

\begin{abstract}
Many bacteria export intracellular calcium using active transporters homologous to the sarco/endoplasmic reticulum $\mathrm{Ca}^{2+}$-ATPase (SERCA). Here we present three crystal structures of $\mathrm{Ca}^{2+}$-ATPase 1 from Listeria monocytogenes (LMCA1). Structures with $\mathrm{BeF}_{3}^{-}$mimicking a phosphoenzyme state reveal a closed state, which is intermediate between the outward-open E2P and the proton-occluded E2- $\mathrm{P}^{*}$ conformations known for SERCA. It suggests that LMCA1 in the E2P state is pre-organized for dephosphorylation upon $\mathrm{Ca}^{2+}$ release, consistent with the rapid dephosphorylation observed in single-molecule studies. An arginine side-chain occupies the position equivalent to calcium binding site I in SERCA, leaving a single $\mathrm{Ca}^{2+}$ binding site in LMCA1, corresponding to SERCA site II. Observing no putative transport pathways dedicated to protons, we infer a direct proton counter transport through the $\mathrm{Ca}^{2+}$ exchange pathways. The LMCA1 structures provide insight into the evolutionary divergence and conserved features of this important class of ion transporters.

(c) 2021 The Authors. Published by Elsevier Ltd. This is an open access article under the CC BY license (http://creativecommons.org/licenses/by/4.0/).
\end{abstract}

\section{Introduction}

$\mathrm{Ca}^{2+}$ regulation is critical for all cells, and therefore also for bacterial cell biology and survival. ${ }^{1}$ Active transporters pump $\mathrm{Ca}^{2+}$ across the membrane to maintain low intracellular $\mathrm{Ca}^{2+}$ concentrations. ${ }^{2}$ Mechanistic details of the calcium transport mechanism have been derived mainly for the sarco/endoplasmic reticulum $\mathrm{Ca}^{2+}$-ATPase (SERCA) and assumed to extrapolate to other calcium pumps. However, $\mathrm{Ca}^{2+}$-ATPases work in a range of different environments across the domains of life, and transport mechanisms must adapt also to specific conditions. To understand how adaptive mechanisms translate sequence variations among $\mathrm{Ca}^{2+}$-ATPases to specific functions, detailed structural information of a more diverse pool of $\mathrm{Ca}^{2+}$ ATPases is helpful.

The gram-positive bacterium Listeria monocytogenes expresses a $\mathrm{Ca}^{2+}$-ATPase (LMCA1), which is homologous to mammalian $\mathrm{Ca}^{2+}$-ATPases. Soil is the natural habitat of Listeria, but they can develop into food borne pathogens, causing listeriosis through infection of 
the bloodstream, spinal cord membranes and brain. LMCA1 extrudes $\mathrm{Ca}^{2+}$ across the bacterial membrane most likely in exchange for a proton. ${ }^{3}$ In contrast to mammalian cells, opportunistic bacteria survive in a range of different external environments. LMCA1 thus allows Listeria to survive in phagosomal compartments of infected host cells, where $\mathrm{Ca}^{2+}$ concentrations can reach millimolar ranges. ${ }^{4}$ Furthermore, LMCA1 is part of a complex regulatory network associated with alkaline $\mathrm{pH}$ tolerance in the intracellular compartments. ${ }^{5}$ LMCA1 is therefore a determinant of Listeria intra-cellular $\mathrm{Ca}^{2+}$ homeostasis and an important model for both calcium regulation in bacteria and $\mathrm{Ca}^{2+}$-ATPase mechanisms.

$\mathrm{Ca}^{2+}$-ATPases comprise the P2A and P2B subtypes of the P-type ATPases, which all share a conserved domain structure and key features of their transport mechanisms, involving formation and breakdown of a phosphoenzyme intermediate. The closest mammalian homologue of LMCA1 is SERCA with $34-39 \%$ sequence identity, depending on the isoform, while the secretory pathway $\mathrm{Ca}^{2+}$-ATPases (SPCA) and plasma-membrane $\mathrm{Ca}^{2+}$-ATPases (PMCA) share $34 \%$ and $27-30 \%$ sequence identity with LMCA1, respectively. ${ }^{3}$ Structurally, $\mathrm{Ca}^{2+}$-ATPases are characterized by a TM (transmembrane) domain consisting of ten helical segments (M1-10), and a cytosolic headpiece defined by three cytosolic domains: $\mathrm{A}$ (actuator), $\mathrm{N}$ (nucleotide) and $\mathrm{P}$ (phosphorylation) domains (Figure 1(A)). These domains move relative to each other during the reaction

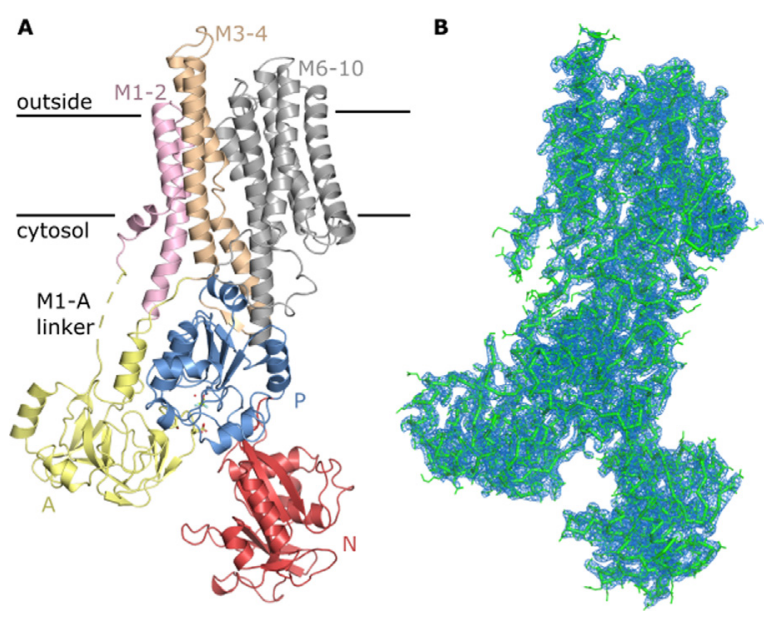

Figure 1. Crystal structure of $\mathrm{G}_{4} \mathrm{E} 2-\mathrm{BeF}_{3}^{-}$at $3.0 \AA$. (A) The crystal structure of $\mathrm{G}_{4} \mathrm{E}_{2}-\mathrm{BeF}_{3}^{-}$comprises the expected P-type ATPase architecture. Represented as cartoon, it is colored according to the domains. The TGES loop, Asp334 and $\mathrm{BeF}_{3}^{-}$are shown as sticks. $\mathrm{Mg}^{2+}$ and $\mathrm{H}_{2} \mathrm{O}$ are shown as spheres. $(\mathrm{B})$ The $2 \mathrm{~F}_{\mathrm{o}}-\mathrm{F}_{\mathrm{c}}$ map is contoured at $1.5 \sigma$ (blue mesh), overlaid with the backbone of $\mathrm{G}_{4} \mathrm{E} 2-\mathrm{BeF}_{3}^{-}$as ribbon and the side chains as lines (green). cycle, resulting in transitions between different functional states. ${ }^{6}$

Although SERCA and LMCA1 share functional and mechanistic properties, they are also different. Per molecule of ATP hydrolyzed, SERCA transports two $\mathrm{Ca}^{2+}$ ions out and counter transports two-three protons, ${ }^{7,8}$ whereas LMCA1 only transports a single $\mathrm{Ca}^{2+}$ ion out and most likely counter transports one proton. ${ }^{3}$ Likewise, SPCA and PMCA only transport a single $\mathrm{Ca}^{2+}$ ion across the membrane per cycle. Of the two $\mathrm{Ca}^{2+}$ binding sites of SERCA, site II is universally conserved among $\mathrm{Ca}^{2+}$-ATPases, while site I is replaced with other functionalities in LMCA $1,{ }^{3}$ PMCA, $^{9}$ and SPCA. ${ }^{10}$ Homology models and mutational studies of LMCA1 have suggested that an arginine (Arg795) occupies the region corresponding to $\mathrm{Ca}^{2+}$ binding site $\mathrm{I}$ in SERCA, and may account for a high pH optimum in LMCA1 ( $\mathrm{pH}$ 8.75-9.5) relative to SERCA ( $\mathrm{pH} \sim 7) .{ }^{3}$ Furthermore, kinetic comparisons suggested that the rate-limiting step is phosphorylation for LMCA1, whereas it is the E1P to E2P transition for SERCA. ${ }^{11}$

The mechanism of $\mathrm{Ca}^{2+}$-ATPases has been described in detail by crystal structures of SERCA trapped by inhibitors at specific intermediate steps of the transport cycle. ${ }^{12}$ A schematic diagram summarizing the functional cycle is given in Figure 2 (A). Overall, ATP hydrolysis drives large structural rearrangements that alter the pump from an inward open (E1) to an outward open state (E2), thereby moving $\mathrm{Ca}^{2+}$ across the membrane. In more detail, binding of $\mathrm{Ca}^{2+}$ to the E1 state leads to ATPdependent phosphorylation of a catalytic aspartate in the $\mathrm{P}$ domain, ${ }^{13,14}$ forming a compact state (E1P). Following phosphoryl transfer, the A domain then undergoes a large rotation, forming the outward-open E2P state. ${ }^{15}$ This occurs via a $\mathrm{Ca}^{2+}$ occluded E2P state and an ADP releasing step at presumably intermediate positions of the $A$ domain that have not yet been observed in crystal structures, although modelled on the basis of solution X-ray scattering data. ${ }^{16}$ Low intracellular ADP concentrations and shielding of the phosphorylated $P$ domain by a conserved TGES loop in the A domain prevent reverse reactions with ADP in the outwardopen E2P state, which is crucial for the directionality of the cycle. Negatively charged residues in the transmembrane ion binding sites of $\mathrm{Ca}^{2+}$-ATPases are protonated, which allows closure of the outward-open ion pathway. This transition is associated with a small rotation of the A domain around the phosphorylation site that places the glutamate side chain of the TGES loop to activate a water molecule for the dephosphorylation reaction by an in-line nucleophilic attack. ${ }^{17-19}$ Dephosphorylation leads to the proton-occluded E2 state stabilized by a hydrophobic cluster that ensures tight association between M2-A and A-M3 linker segments and the $P$ domain with a $\mathrm{K}^{+}$site..$^{20,21}$ Proton release from the ion binding sites to the cytosolic side allows the 

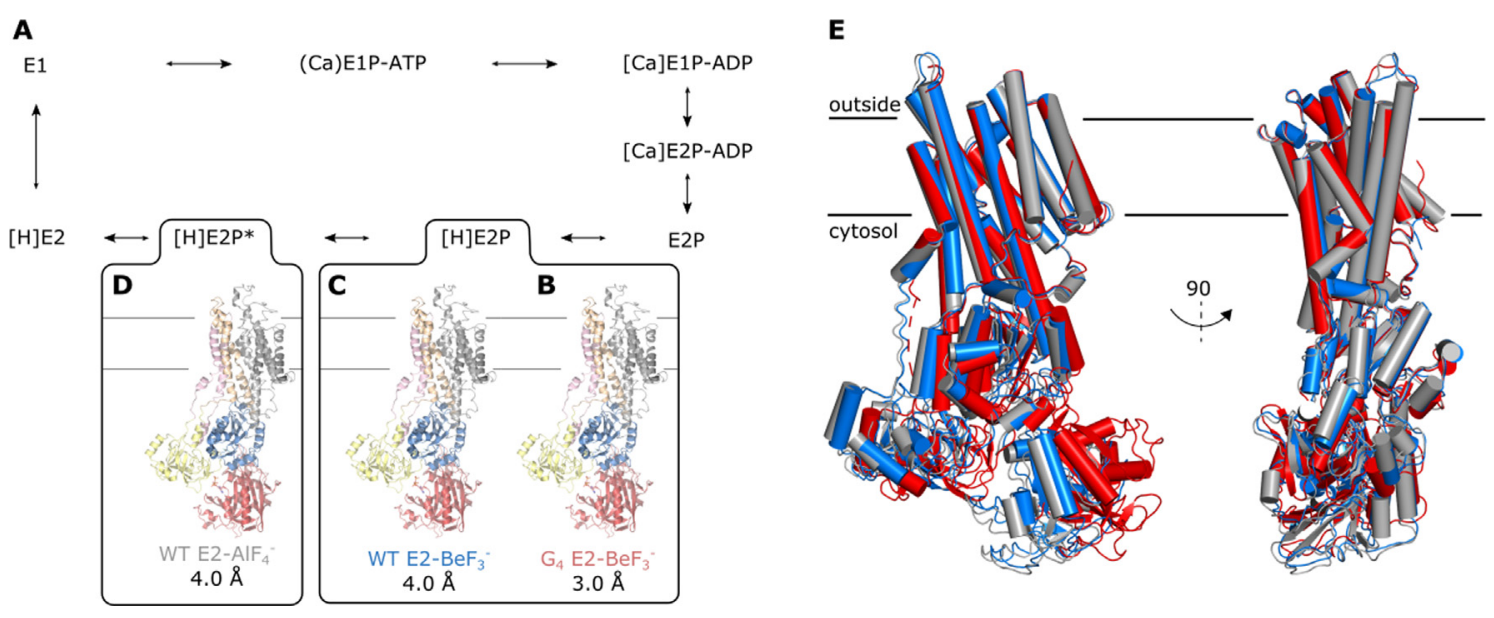

Figure 2. LMCA1 structures adopt proton-occluded E2 states. (A) The reaction cycle of LMCA1 with crystal structures represented by (B) $\mathrm{G}_{4} \mathrm{E} 2-\mathrm{BeF}_{3}^{-}$, (C) WT E2-BeF- and (D) WT E2-AIF- The structures are colored according to the different domains. (E) An alignment of WT E2-AlF ${ }_{4}^{-}$(light grey), WT E2-BeF-' (blue) and $\mathrm{G}_{4} \mathrm{E}_{2}-\mathrm{BeF}_{3}^{-}$ (red). All the structures are aligned by transmembrane helices M7-10. A different angle of the cytosolic headpiece relative to the TM domain is observed for the $G_{4}$ mutant.

pump to adopt the inward-open E1 state and again engage in $\mathrm{Ca}^{2+}$ binding.

Previously, LMCA1 was crystallized in a $\mathrm{Ca}^{2+}$ free state similar to the dephosphorylation intermediate (denoted $\mathrm{E}^{-}-\mathrm{P}^{*}$ ) using $\mathrm{AlF}_{4}^{-}$as a mimic of a pentavalent (trigonal bipyramidal) transition state of dephosphorylation. The structure was partially determined at $4.3 \AA$ resolution, ${ }^{22}$ but did not permit any detailed analysis. We have also investigated LMCA1 using single-molecule FRET (smFRET). ${ }^{11,23}$ These data showed that LMCA1 could be paused in E2P states by introduction of a four glycine insert into the A-M1 linker $\left(G_{4}\right.$ mutant, identified from SERCA $\left.{ }^{24}\right)$ and most noteworthy, an occluded E2P state preceding $\mathrm{Ca}^{2+}$ release was observed. $\mathrm{BeF}_{3}^{-}$can be coordinated by the Asp side chain in the phosphorylation site of P-type ATPases, and thereby stabilize a complex mimicking an E2P phosphoenzyme. The smFRET data showed that LMCA1 can be inhibited in mediating dephosphorylation both by forming complex with $\mathrm{BeF}_{3}^{-}$and by an $\mathrm{E} 167 \mathrm{Q}$ mutant form that targets the TGES motif. ${ }^{11}$

We have further explored crystallization of LMCA1 and present here three structures stabilized by metal fluorides: a $G_{4}$ mutant structure with $\mathrm{BeF}_{3}^{-}$at $3.0 \AA$ resolution, and two wild-type structures at $4.0 \mathrm{~A}$ resolution, including a structure with $\mathrm{BeF}_{3}^{-}$and an improved structure with $\mathrm{AlF}_{4}^{-}$.

\section{Results}

To gain new insight into the functional effect of the $\mathrm{G}_{4}$ mutant, we crystallized it in the $\mathrm{E}_{2}-\mathrm{BeF}_{3}^{-}$ form and obtained well diffracting crystals at $3.0 \AA$ (Figure 1, Table S1). The asymmetric unit contained 8 molecules related by non-crystallographic symmetry (Figure S1). The refined structure of LMCA1 derived from this crystal form was then used as a molecular replacement model to determine details of LMCA1 dephosphorylation by the structures of wild-type LMCA1 in the E2-BeF- and E2-AlF- forms at $4.0 \AA$ resolution.

\section{Overall conformation}

All three crystal structures show the overall structure expected for a P-type ATPase with three cytosolic domains connected to a membrane domain with 10 transmembrane segments (Figure 1(A) and 2(B)-(D)). Surprisingly, WT E2$\mathrm{BeF}_{3}^{-}$and WT E2-AIF- adopt the same overall conformation, and the configuration of the cytosolic domains is similar to E2- $\mathrm{P}^{*}$ like conformations of SERCA in complex with $\mathrm{AIF}_{4}^{-}$ (Figure S2(A) and Table S2). Like the WT constructs, the $\mathrm{E} 2-\mathrm{BeF}_{3}^{-}$form of the $\mathrm{G}_{4}$ mutant adopts a conformation more similar to SERCA E2$\mathrm{AlF}_{4}^{-}$(Table S2), even though the orientation of the cytosolic domains looks more similar to SERCA $\mathrm{E} 2-\mathrm{BeF}_{3}^{-}$(Figure S2(B)). This can be explained by the position of the cytosolic domains relative to the $\mathrm{TM}$ being tilted in $\mathrm{G}_{4} \mathrm{E}_{2}-\mathrm{BeF}_{3}^{-}$(Figure 2(E)). However, the $A$ domain makes different crystal contacts in WT and $\mathrm{G}_{4}$ structures (Figure S3), so changes in the domain orientation should be interpreted with caution.

The electron density map reveals no indication of $\mathrm{Ca}^{2+}$ bound at the site of the ion binding site in $\mathrm{G}_{4}$ $\mathrm{E} 2-\mathrm{BeF}_{3}^{-}$even though the crystallization buffer contained $2 \mathrm{mM} \mathrm{Ca}^{2+}$, thus all three structures appear to represent occluded states with a closed extracellular pathway and presumably a protonated ion binding site (Figure 2(B)-(D)). In 
the following, we will focus on the structure with the highest resolution $\left(\mathrm{G}_{4} \mathrm{E} 2-\mathrm{BeF}_{3}^{-}\right)$when discussing detailed structural features.

The structures stabilized by $\mathrm{BeF}_{3}^{-}$and $\mathrm{AlF}_{4}^{-}$inform on transitions associated with extracellular $\mathrm{Ca}^{2+}$ release and protonation coupled to dephosphorylation. For the WT E2-BeF- and E2$\mathrm{AlF}_{4}^{-}$structures the resolution limited analysis of the side chain orientations, however the side chains are still modeled. The $\mathrm{N}$ domain is poorly defined in the electron density maps of the WT structures (Figure S4), and thus likely to be flexible. The $\mathrm{G}_{4} \mathrm{E}_{2}-\mathrm{BeF}_{3}^{-} \mathrm{MR}$ model provided the overall fold of the $\mathrm{N}$ domains and most helices are defined; thus, the $\mathrm{N}$ domains are still modeled despite the poor density. The asymmetric unit of the $\mathrm{G}_{4} \mathrm{E}_{2}-\mathrm{BeF}_{3}^{-}$crystal contains eight copies of the protein, where four have $\mathrm{N}$ domains that are well defined in electron density, while the other four exhibit again weak density for the $\mathrm{N}$ domains, indicating flexibility (Figure S5). The flexibility can be related to a minimal role of the $\mathrm{N}$ domain in the dephosphorylation half-cycle. The relative position of the $\mathrm{N}$ domain of the $\mathrm{G}_{4} \mathrm{E}_{2}-\mathrm{BeF}_{3}^{-}$structure differs from that of the WT structure, but the $\mathrm{P}$ and $A$ domains maintain the same relative configuration, only somewhat tilted relative to the TM domain (Figure 3(A)). Moreover, the A-M1 linker region of the $G_{4}$ mutant appears to be flexible, which uncouples the $A$ domain and the position of the entire cytosolic headpiece relative to the TM domain that is tilted in the $G_{4}$ mutant compared to the WT structures (Figure 2(E)).

\section{Interaction between the TGES loop in the A domain and the $\mathbf{P}$ domain}

The A-P domain interface is critical for dephosphorylation, which is coupled to protonation and occlusion of the extracellular ion exchange pathway. Besides the TGES loop interacting with the phosphorylation site, the interface is also stabilized by Asp186 of the A domain forming an ionic interaction with Arg598 of the P-domain. Only the $\mathrm{G}_{4} \mathrm{E}_{2}-\mathrm{BeF}_{3}^{-}$structure allows proper refinement of side chain orientations, but similarity of local backbone conformations of the available LMCA1 structures hint at generally preserved interactions in all three structures. In SERCA, the A-P domain interface is connected by several ionic bonds referred to as an 'electrostatic catch' centered on Arg198 in the A domain, which is also important for the dephosphorylation rate of SERCA. ${ }^{25}$ This residue is not conserved in LMCA1, which indicates that different interactions control LMCA1 dephosphorylation. Despite the fewer interactions defining the A$P$ domain interface in LMCA1, smFRET experiments have shown that the pump dephosphorylates rapidly, indicating that the required interactions form quickly. ${ }^{11}$ With fewer interactions involved, a catalytically competent state may be reached faster in LMCA1.

The two metal fluorides used as phosphate analogues trap LMCA1 in states before and during dephosphorylation. In all three structures presented, the A domain is fully rotated and associated with the $\mathrm{P}$ domain in a position that corresponds to the dephosphorylation state of SERCA $\left(E 2-P^{*}\right)$ (Figure 3(A)). In $\mathrm{G}_{4}$ E2-BeFTGES loop orients the side chain of Glu167 (Glu183 in SERCA) towards the phosphate analogue, i.e. poised for dephosphorylation (Figure 3(B)). Position of Glu167 side chain was confirmed by an omit annealed Fo-Fc map (Figure S6). In SERCA, the calcium-free $\mathrm{BeF}_{3}^{-}$ complex adopts an outward-open E2P state, where the TGES loop shields the phosphorylated aspartate and the Glu183 side chain (corresponding to LMCA1 Glu167) points away from the phosphorylation site (Figure 3(C)). SERCA only adopts an occluded, dephosphorylation state in the $\mathrm{AlF}_{4}^{-}$complex,
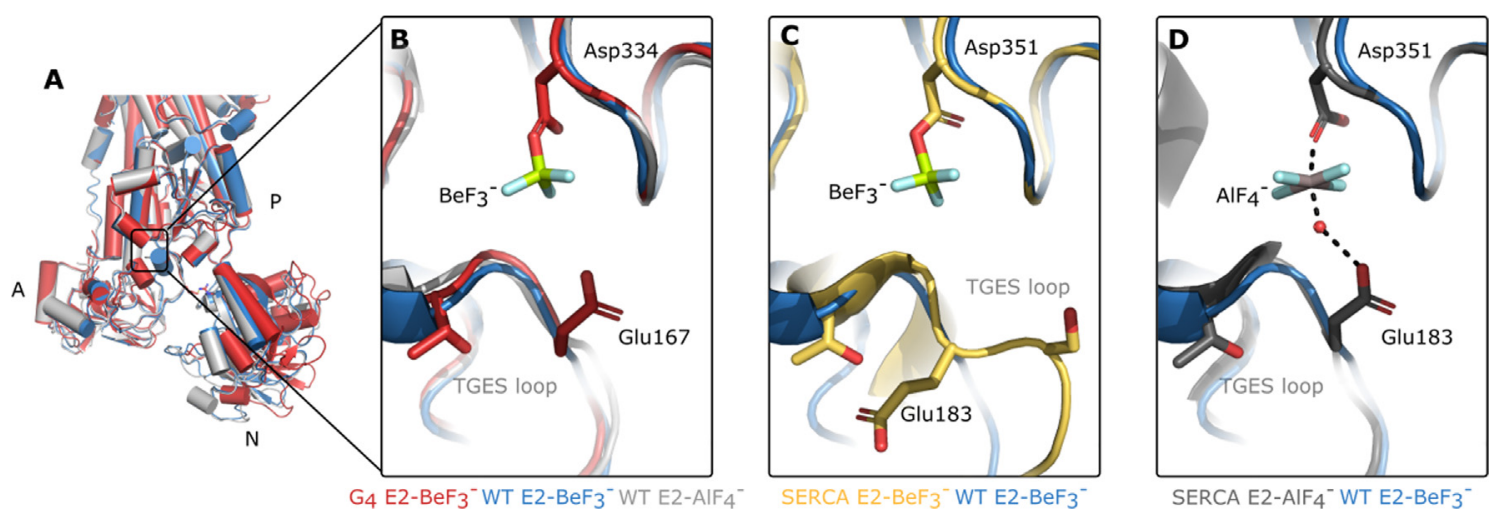

Figure 3. The TGES loop in the E2P state is pre-positioned to catalyze dephosphorylation in LMCA1, but not in SERCA. (A) LMCA1 WT E2-AIF- (light grey), WT E2-BeF-- (blue) and $\mathrm{G}_{4}$ E2-BeF 3 (red). (B) Zoom of the TGES loop of (A). The TGES loop of LMCA1 WT E2-BeF 3 aligned with (C) SERCA E2-BeF- (pdb: 3b9b) (orange) and (D) SERCA $\mathrm{E}_{2}-\mathrm{AlF}_{4}^{-}$(pdb: 3b9r) (dark grey). All the structures are aligned by the $\mathrm{P}$ domain. 
where Glu183 approaches the phosphorylation site and activates the water molecule that acts as the nucleophile in the hydrolysis (Figure 3(D)). Since LMCA1 $\mathrm{E}_{2}-\mathrm{BeF}_{3}^{-}$and $\mathrm{E}_{2}-\mathrm{AlF}_{4}^{-}$complexes have similar domain arrangements as SERCA E2-AIF-, no large-scale conformational changes seem necessary to initiate the phosphatase activity following $\mathrm{Ca}^{2+}$ release (Figure $3(\mathrm{~B})$ ). It suggests that LMCA1 adopts a state preactivated for dephosphorylation upon $\mathrm{Ca}^{2+}$ release.

\section{Ion pathways and binding sites}

In LMCA1, the $\mathrm{Ca}^{2+}$ release pathway is closed in all structures, sealed with an ionic bond between Asp702 (loop M5-6) and Arg261 (M3) and van der Waal interactions. These residues are not conserved in SERCA. The closed extracellular pathway in LMCA1 is further supported by a hydrophobic cluster consisting of Leu107, Met110, Met163, Leu164, Ile215, Val625 and Val646 associating $\mathrm{M} 2, \mathrm{M} 3$ and the $\mathrm{P}$ domain at the cytosolic side; this is however conserved in SERCA and represents a characteristic feature of proton-occluded E2 structures. ${ }^{15,19,26}$

LMCA1 transports a single $\mathrm{Ca}^{2+}$ ion and likely counter transports a single proton per hydrolyzed ATP. ${ }^{3}$ Alignment of the crystal structures show that the $\mathrm{Ca}^{2+}$ binding site of LMCA1 is similar to site II in SERCA (Figure 4). Arg795 on the other hand replaces the $\mathrm{Ca}^{2+}$-coordinating Glu908 found in SERCA site I as proposed previously. ${ }^{3}$ The other ion-coordinating residue of site $\mathrm{I}$ in SERCA (Glu771) 27 is replaced by Ala691 in LMCA1. The short side chain leaves space for the side chain of Arg795 to extend into the core of the LMCA1 TM

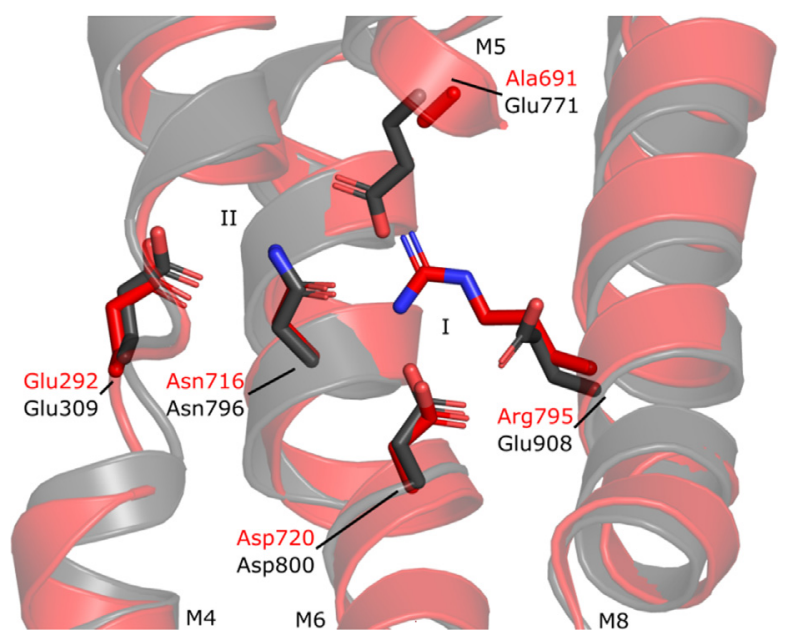

Figure 4. Binding site I of SERCA is not conserved in LMCA1. Alignment between LMCA1 G $\mathrm{E}_{4}-\mathrm{BeF}_{3}^{-}$(red) and SERCA E2-AlF- (pdb: 3b9r) (dark grey). Relevant $\mathrm{Ca}^{2+}$-coordinating residues are shown as sticks. The structures are aligned by the residues shown as sticks. SERCA $\mathrm{Ca}^{2+}$ binding site I and II are indicated. domain and in effect substitute for $\mathrm{Ca}^{2+}$ at the site corresponding to site I in SERCA.

SERCA has two proposed proton pathways: a luminal entry pathway ${ }^{28}$ and a C-terminal cytosolic release pathway. ${ }^{29}$ These are in addition to the luminal $\mathrm{Ca}^{2+}$ release pathway between $\mathrm{M} 1 / \mathrm{M} 2$, $\mathrm{M} 3 / \mathrm{M} 4$ and $\mathrm{M} 5 / \mathrm{M6}^{15}$ and the N-terminal cytosolic $\mathrm{Ca}^{2+}$ entry pathway between $\mathrm{M} 1, \mathrm{M} 2$, and $\mathrm{M} 3{ }^{13}$ The proposed luminal proton pathway is a narrow water channel lined by hydrophilic residues in M5 and $M 7$, leading protons to binding site $I$ of SERCA. ${ }^{28}$ The corresponding part of LMCA1 is mainly hydrophobic, indicating that protons cannot enter through such a pathway.

The C-terminal cytosolic pathway, which is proposed to lead protons from binding site I to the cytosol in SERCA, is also different in LMCA1 (Figure 5). In SERCA, it consists of a narrow hydrated wire between M5, M7, M8 and M10, and the hydrophilic side chains of the residues involved in the water network orient into the cavity (Figure $5(\mathrm{~A})$ and $(\mathrm{B})$ ). ${ }^{29}$ Although identification of water molecules in LMCA1 is restricted by the resolution of the structures, the nature of the corresponding residues lining the pathway can indicate if a hydrated wire is also present in LMCA1. In fact, the majority of water coordinating residues in the cytosolic pathway in SERCA are different in LMCA1 (Figure 5(C) and (E)). For example, Tyr837, Asn914 and Asn981 in SERCA correspond to Gly761, Ala801 and Met868 in LMCA1. The pathway lacks almost entirely polar groups to make a tight interaction network with water molecules. One exception is Arg760 in M7 that extends into the pathway with the aliphatic part occupying the space corresponding to the hydrated cavity in SERCA (Figure 5(D)). Essentially, M7 is shifted compared to SERCA and Arg760 sterically blocks the pathway in LMCA1. In SERCA, the equivalent position in M7 is Arg836 (Figure 5(D)). However, this arginine surrounds and coordinates the water molecules in the cavity. Altogether, LMCA1 does not appear to have a continuous water wire that can lead protons from the $\mathrm{Ca}^{2+}$ site to the cytosol.

\section{Transport site protonation}

$\mathrm{Ca}^{2+}$ binding sites in $\mathrm{Ca}^{2+}$-ATPases are also sites for proton counter transport by protonation of glutamate and aspartate residues involved in $\mathrm{Ca}^{2+}$-coordination. In SERCA, the two glutamates (Glu908 and Glu771) in site I are assumed to be protonated from the lumen in the $\mathrm{Ca}^{2+}$ free states and shuttle proton across the membrane in the E2 to $\mathrm{E} 1$ transition. ${ }^{30}$ These proton-transporting side chains are not present in LMCA1, so how are protons then counter transported? Proton counter transport is observed in $\mathrm{LMCA} 1^{3}$ and low $\mathrm{pH}$ stabilizes the E2 states of LMCA1 $1{ }^{11}$ suggesting that the ion binding site is indeed protonated in the $\mathrm{Ca}^{2+}$-free state. Unless at very high resolution, crystal structures do not reveal protonation directly, but the 

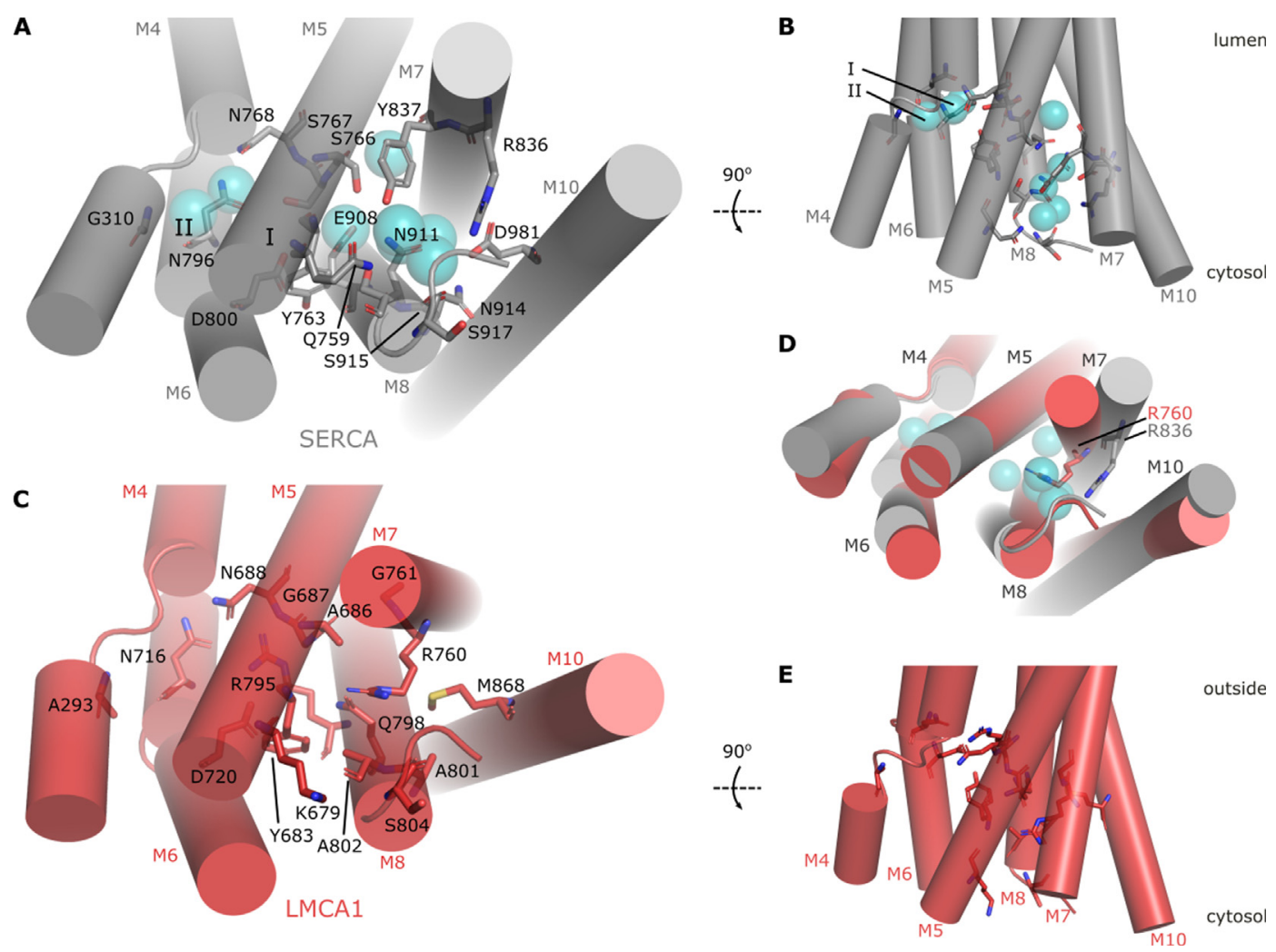

Figure 5. The C-terminal cytosolic pathway leading to site I in SERCA is different in LMCA1. (A, B) In SERCA E2$\mathrm{AlF}_{4}^{-}$(pdb: 3n5k) (grey), the C-terminal pathway forms a continuous hydrophilic cavity that leads to ion binding site I. $(\mathrm{C}, \mathrm{E}) \mathrm{G}_{4} \mathrm{E}_{2}-\mathrm{BeF}_{3}^{-}$(red), many of the residues lining the cytosolic cavity are small and hydrophobic. (D) Alignment of LMCA1 and SERCA shows that M7 is shifted and Arg760 extends into the cavity in LMCA1, whereas Arg836 coordinates the water molecules in SERCA. Water molecules are shown as blue spheres. Only M4-8 and M10 are shown, and the cytosolic domains are omitted for clarity. Residues interacting with water in SERCA E2-AIF- ${ }_{4}$ are shown as sticks, as well as the corresponding residues in $\mathrm{G}_{4} \mathrm{E}_{2}-\mathrm{BeF}_{3}^{-}$. SERCA Ca ${ }^{2+}$ binding site I and II are indicated.

structure of the ion binding site leaves only two potential candidates that can be reversibly protonated: Glu292 and Asp720 (corresponding to Glu309 and Asp800 in SERCA) (Figure 4). To investigate the protonation state of these residues, we estimated $p K_{a}$ values based on the $G_{4}$ E2$\mathrm{BeF}_{3}^{-}$crystal structure using PROPKA. ${ }^{31}$ The $\mathrm{p} K_{\mathrm{a}}$ values of Glu292 and Asp720 are estimated at 8.9 and 6.2, respectively, which suggests that Asp720 remains negatively charged in the E2P state, whereas Glu292 becomes protonated and therefore likely is the site for proton counter transport in LMCA1.

\section{Discussion}

We have determined three crystal structures of LMCA1 in E2-BeF- and E2-AlF 4 bound forms representing the E2P and E2-P* transition state of dephosphorylation. In all cases, the TGES loop is pre-positioned for dephosphorylation and the ion binding sites in the TM domain appear to be buried with an occluded proton. It should be noted that occlusion has a slightly different meaning for protons than e.g. $\mathrm{Ca}^{2+}$, as it is difficult to experimentally demonstrate proton occlusion. Furthermore, a crystal structure only provides a single snapshot of a protein, and it is likely that protein dynamics will allow proton access to a certain extent. In the following, we describe a state as proton occluded, if the ion binding site is closed to direct solvent access.

The structures reveal the architecture of the single $\mathrm{Ca}^{2+}$ binding site of LMCA1 in the protonated state and suggest a simpler mechanisms of proton counter transport compared to SERCA. The structural comparison suggests that residues that constitute the active $\mathrm{Ca}^{2+}$ binding site (site II in SERCA) are conserved across a billion years of divergent evolution, but also that LMCA1 and SERCA are individually finetuned for different physiological environments. Insertion of four glycines in the linker between the A domain and M1 of LMCA1 causes the enzyme to pause in a $\mathrm{Ca}^{2+}$-occluded E2P state. ${ }^{11,24}$ The $\mathrm{BeF}_{3}^{-}$-stabilized form of this mutant showed no bound $\mathrm{Ca}^{2+}$ and represents a proton-occluded pre-state of dephosphorylation, similar to structures of the wild-type enzyme with $\mathrm{BeF}_{3}^{-}$and $\mathrm{AlF}_{4}^{-}$, 
although with slightly altered angle of the cytosolic domains relative to the TM domain.

\section{Interdomain interactions account for the fast rate of dephosphorylation}

A smFRET study showed that relative rates of partial reactions differ between $\mathrm{LMCA} 1^{11}$ and SERCA. ${ }^{25}$ LMCA1 is rate-limited by phosphorylation, dephosphorylates rapidly, and thus predominantly accumulates in E1 states during steadystate pumping. ${ }^{11}$ But which structural differences between SERCA and LMCA1 account for the different dephosphorylation rates? Crystal structures of $\mathrm{E} 2-\mathrm{BeF}_{3}^{-}$and E2-AIF ${ }_{4}^{-}$complexes show that LMCA1 and SERCA adopt different conformations when stabilized by the same metal fluorides as phosphate analogues. SERCA E2-BeF ${ }_{3}^{-}$forms an outwardopen E2P state with the TGES loop shielding the phosphate analogue. However, the LMCA1 E2$\mathrm{BeF}_{3}^{-}$and E2-AlF-- complexes adopt a similar conformation to the proton-occluded SERCA E2-AIFThe LMCA1 structures all have the TGES loop in a position, where the glutamate side chain can orient a water molecule for in-line attack on the phosphorylated aspartate of the $\mathrm{P}$ domain (Figures $3(\mathrm{~B})$ and S5). An outward-open E2P state and a protonoccluded E2- $P^{*}$ intermediate state are likely in rapid equilibrium, but the structural studies point to differences for LMCA1 and SERCA. SERCA changes its conformation to induce dephosphorylation in response to uptake of two-three protons through separate proton pathways, and the outward-open E2P state is stabilized by many interactions - these mechanisms can be targeted by regulation. In LMCA1 however, protonation, occlusion and activation of the dephosphorylation site is favored directly upon $\mathrm{Ca}^{2+}$ release.

\section{Binding sites for one or two $\mathrm{Ca}^{2+}$}

While SERCA transports two $\mathrm{Ca}^{2+}$ per cycle, other mammalian P-type ATPases such as a PMCA and SPCA transport only a single $\mathrm{Ca}^{2+}$ ion, like LMCA1. While LMCA1 has a similar level of sequence identity to SERCA and PMCA, it may thus be mechanistically more similar to PMCA. A decreased transport stoichiometry increases the driving force for ion transport, but removes regulatory mechanisms enacted by cooperative binding as in SERCA, and likely it represents adaptation to different physiological roles. A low resolution cryo-EM structure of PMCA was determined, ${ }^{9}$ which allows further comparison between the single $\mathrm{Ca}^{2+}$ binding site of mammalian and bacterial $\mathrm{Ca}^{2+}$-ATPases. Similar to LMCA1, PMCA has an ion binding site that corresponds to site II in SERCA. In LMCA1, the missing calcium binding site $I$ is filled with a positive charge from an arginine residue at a position corresponding to Glu908 in SERCA - a trait that is shared among a range of bacterial $\mathrm{Ca}^{2+}$-ATPases. ${ }^{3}$ In PMCA, the corresponding position is occupied by a neutral glutamine ${ }^{9}$ while the residue corresponding to Glu771 in SERCA is an alanine for PMCA. No structure has been reported for the SPCA family, but the ion binding site composition can be predicted from homology modeling. ${ }^{32}$ No compensating positive charge is found in SPCA, but an aspartic acid occupies the position of the Arg795 in LMCA1, and it may be a unique protonation site. Instead, the calcium site I of SPCA is rendered unfunctional by an alanine in the position of SERCA Glu771, as observed also for LMCA1 and PMCA. This shows that $\mathrm{Ca}^{2+}$ ATPases have evolved different mechanisms to alter the transport stoichiometry, and the charge replacement strategy found in LMCA1 is not a unique strategy for stabilizing a non-functional ion binding site.

\section{Mechanism of proton counter transport}

The altered ion binding site in LMCA1 suggests an altered mechanism of proton counter transport. Our crystal structures indicate that Glu292 (Glu309 in $\mathrm{Ca}^{2+}$ site II of SERCA) has an elevated $\mathrm{pK}_{\mathrm{a}}$, which is the functional requirement for a protonation site at physiological or even alkaline $\mathrm{pH}$. In all E2-like states of SERCA, a C-terminal water wire connects Glu309 to the cytosol, ${ }^{29}$ and it is not believed to counter transport protons. For Glu292 in LMCA1 to participate in counter transport, the protonation must occur from the extra-cellular side. LMCA1 does not seem to have an additional proton pathway, and protonation must occur directly through the calcium ion exit pathway. LMCA1 thus resembles the $\mathrm{P} 3$-type $\mathrm{H}^{+}$-ATPases that also seem to feature single entry and exit pathways. ${ }^{33,34}$ This direct mechanism of protonation may relate to the fact that only a single site is involved, thus reducing the need for rapid neutralization of the empty binding site and bifurcated ion pathways. ${ }^{35}$ Furthermore, protonation of Glu309 in SERCA is believed to be crucial for closure of the luminal pathway and transition to the proton-occluded state mimicked by E2-AIF- ${ }_{4}{ }^{15}$ In an unpredictable, and potentially hostile, external environment, an outwardopen state is vulnerable to spurious interactions with compounds. Rapid occlusion and dephosphorylation may also be an evolutionary mechanism of protection for outside-facing pumps.

\section{The proton-occluded E2P state precedes dephosphorylation}

A molecular dynamics study of SERCA proposed that after $\mathrm{Ca}^{2+}$ release, the pump transitions into a proton-occluded E2P state with a closed luminal pathway before dephosphorylation. ${ }^{18} \mathrm{~A}$ key prediction of this study was the formation of an intermediate between known crystal structures. ${ }^{18}$ This predicted intermediate would correspond to the occluded structure stabilized by $\mathrm{BeF}_{3}^{-}$that is observed here. The direct observations of such an 
intermediate from LMCA1 crystal structures is consistent with these simulations. Such an intermediate may have been observed also earlier in the crystal structure of SERCA in an E2- $\mathrm{BeF}_{3}^{-}$form with the inhibitor thapsigargin. ${ }^{36}$ This structure features a proton-occluded TM domain and cytosolic domains in the E2P state with the phosphorylation site shielded by the TGES loop. Indeed, the LMCA1 structures determined here are more consistent with the thapsigargin bound SERCA and especially in the orientation of M1-2.

\section{A-M1 linker couples the cytosolic domains and TM}

The structure of $\mathrm{G}_{4} \mathrm{E} 2-\mathrm{BeF}_{3}^{-}$aligns well to WT E2$\mathrm{BeF}_{3}^{-}$in the TM domain, but the cytosolic domains are positioned differently (Figure 2(E)). In $\mathrm{G}_{4}$ E2$\mathrm{BeF}_{3}^{-}$, the all the cytosolic domains are tilted compared to WT E2- $\mathrm{BeF}_{3}^{-}$and the $\mathrm{N}$ domain separates more from the A domain. In fact, we noted two different clusters of conformations in the eight copies in the asymmetric unit (Figure S1(A)), where the orientation of the cytosolic domains varies slightly relative to the TM domain (Figure $\mathrm{S} 1(\mathrm{~B})$ ). This suggests that the entire cytosolic headpiece is subject to rigid body movements, and that extension of the A-M1 linker alters the interdomain dynamics.

In SERCA, the $\mathrm{G}_{4}$-insertion in the A-M1 linker extends the lifetime of a $\mathrm{Ca}^{2+}$-occluded $\mathrm{E}_{2}-\mathrm{BeF}_{3}^{-}$ intermediate state preceding $\mathrm{Ca}^{2+}$ release, ${ }^{24}$ and this state can be entered both through a forward and a reverse reaction. In LMCA1, sMFRET data also showed that the $G_{4}$ mutant stabilized the $\mathrm{Ca}^{2+}$-occluded E2P state under pumping conditions, albeit only briefly. We crystallized the LMCA1 $\mathrm{G}_{4}$ mutant form in the $\mathrm{E}_{2}-\mathrm{BeF}_{3}$ form in the presence of $\mathrm{Ca}^{2+}$, but observed no bound $\mathrm{Ca}^{2+}$. The smFRET study of LMCA1 offers a rationale for this, as $\mathrm{Ca}^{2+}$ and ADP release was observed to be practically irreversible for LMCA1. ${ }^{11}$ Thus, despite the presence of high $\mathrm{Ca}^{2+}$ concentrations, LMCA1 apparently cannot enter this $\mathrm{Ca}^{2+}$-occluded E2P intermediate in the reverse direction, and the $G_{4}$ mutant thus crystallized in a $\mathrm{Ca}^{2+}$-free form. Unlike the WT complexes, we did not observe density for the central part of the $\mathrm{G}_{4}$-extended A-M1 linker in the crystal structure of $\mathrm{G}_{4} \mathrm{E} 2-\mathrm{BeF}_{3}^{-}$, showing that indeed it gains flexibility and probably weakens mechano-chemical coupling.

\section{Conclusion}

$\mathrm{Ca}^{2+}$-ATPases are ubiquitous throughout all domains of life. With structures of a prokaryotic $\mathrm{Ca}^{2+}$-ATPase, we illustrate how ATPases have diverged mechanistically and structurally throughout evolution including changes in the transport stoichiometry. We observe an intermediate of dephosphorylation that was predicted earlier by molecular dynamics simulations. Future studies should illuminate mechanistic differences of single-site $\mathrm{Ca}^{2+}$ ATPases to SERCA-type pumps with cooperative sites, where LMCA1 serves as a convenient surrogate for the mammalian single-site transporters.

\section{Materials and Methods}

\section{Mutagenesis}

LMCA1 $\mathrm{G}_{4}$ : Four glycines between residue K44 and D45 in LMCA1-pET-22b were introduced using the QuickChange mutagenesis kit (Agilent Technologies) and verified by Sanger sequencing (Eurofins, MWG).

\section{Expression and purification}

LMCA1-pET-22b or $\mathrm{G}_{4}$-LMCA1-pET-22b, containing a ten-histidine tag and a Tobacco Etch Virus (TEV) protease site in the C-terminus was expressed and purified according to the protocol described in Faxen et al. $^{3}$ The solubilized membranes were either applied to a pre-packed HisTrap HP column (GE Healthcare) or incubated with $1 \mathrm{~mL}$ $\mathrm{Ni}^{2+}$ slurry (Ni-sepharose 6 Fast Flow, GE Healthcare) per $1 \mathrm{~L}$ expression medium for $1 \mathrm{~h}$, which was then packed into an XK-16 column (GE Healthcare). The protein was eluted with $150 \mathrm{mM}$ imidazole in buffer C $(50 \mathrm{mM}$ Tris- $\mathrm{HCl}, 200 \mathrm{mM} \mathrm{KCl}$, $20 \% \mathrm{v} / \mathrm{v}$ glycerol, $1 \mathrm{mM} \mathrm{MgCl}_{2}, 5 \mathrm{mM} \beta$ mercaptoethanol (BME), $0.25 \mathrm{mg} / \mathrm{mL}$ octaethylene glycol monododecyl ether $\left.\left(\mathrm{C}_{12} \mathrm{E}_{8}\right), \mathrm{pH}=7.6\right)$, and then digested with TEV protease $(1 \mathrm{mg}$ per $20 \mathrm{mg}$ protein), while dialyzed against buffer $\mathrm{C}$ without imidazole (1:100). The digest was applied to a gravityflow $\mathrm{Ni}^{2+}$ column, and the flow-through collected and concentrated to $\sim 10 \mathrm{mg} / \mathrm{mL}$ using Vivaspin $(\mathrm{MWCO}=50 \mathrm{kDa})$. Size-exclusion chromatography was then carried out using a Superdex 200 Increase 10/300 GL column (GE healthcare) equilibrated in $100 \mathrm{mM}$ MOPS, $80 \mathrm{mM} \mathrm{KCl}, 20 \% \mathrm{v} / \mathrm{v}$ glycerol, $3 \mathrm{mM} \mathrm{MgCl}$, $5 \mathrm{mM}$ BME, $0.25 \mathrm{mg} / \mathrm{ml} \mathrm{C}_{12} \mathrm{E}_{8}$, $\mathrm{pH}=6.8$. Finally, the collected LMCA1 was concentrated to $\sim 10 \mathrm{mg} / \mathrm{mL}$.

\section{Relipidation, crystallization and data collection}

$\mathrm{G}_{4} \mathrm{E}_{2}-\mathrm{BeF}_{3}^{-}$was relipidated following the HiLiDe approach. ${ }^{17}$ A $5 \mathrm{~mL}$ glass tube was rinsed with a flow of $\mathrm{N}_{2}$ gas to remove the $\mathrm{O}_{2} .0 .3 \mathrm{mg} \mathrm{1,2-}$ dioleoyl-sn-glycero-3phosphatidylcholine (DOPC) solubilized in $\mathrm{CHCl}_{3}$ was added to the glass tube, and the $\mathrm{CHCl}_{3}$ was evaporated with $\mathrm{N}_{2}$. Subsequently, $100 \mu \mathrm{L} \mathrm{G}_{4} \mathrm{LMCA} 1$ was added, and the tube was sealed with parafilm and stirred at $50 \mathrm{rpm}$ using microstirring bars at $4{ }^{\circ} \mathrm{C}$ overnight. Insoluble material was removed by centrifugation at $190.000 \mathrm{~g}$ for $10 \mathrm{~min}$, and the supernatant was supplemented with $2 \mathrm{mM} \mathrm{CaCl}_{2}$ and a premix of $0.1 \mathrm{mM} \mathrm{BeSO}_{4}$ 
and $0.5 \mathrm{mM} \mathrm{NaF} .1 \mu \mathrm{L}$ of the protein solution was mixed with $1 \mu \mathrm{L}$ of reservoir solution (18\% PEG2000, 8\% glycerol, 8\% MPD, $100 \mathrm{mM} \mathrm{MgCl}$ and $50 \mathrm{mM}$ Tris, $\mathrm{pH}=7.2$ ) on a cover slip and equilibrated against $500 \mu \mathrm{L}$ of the reservoir solution using hanging drop vapor diffusion method. It was sealed with immersion oil (Merck) and equilibrated at $19{ }^{\circ} \mathrm{C}$. Crystals were mounted in loops from mother liquid and flash frozen in liquid $\mathrm{N}_{2}$. A complete data set was collected on the P13 beamline at PETRA III radiation source of Deutches Elektronen-Synchrotron (DESY) using a PILATUS $6 \mathrm{M}$ detector.

WT $\mathrm{E} 2-\mathrm{BeF}_{3}^{-}$was relipidated using the same approach as for $\mathrm{G}_{4} \mathrm{E}_{2}-\mathrm{BeF}_{3}^{-}$. $0.3 \mathrm{mg} \mathrm{DOPC}$ and $0.75 \mathrm{mg} \mathrm{C}_{12} \mathrm{E}_{8}$ solubilized in $\mathrm{H}_{2} \mathrm{O}$ were added to $100 \mu \mathrm{L}$ protein. After centrifugation, LMCA1 was treated with $2 \mathrm{mM}$ ethylene glycol-bis( $\beta$ aminoethyl ether)- $N, N, N, N$-tetraacetic acid (EGTA) and $1 \mathrm{mM} \mathrm{BeSO}_{4}$ premixed with $5 \mathrm{mM}$ $\mathrm{NaF} .1 \mu \mathrm{L}$ of protein solution was mixed with $1 \mu \mathrm{L}$ of modified reservoir solution (7\% PEG6000, 3\% t$\mathrm{BuOH}, 100 \mathrm{mM} \mathrm{LiSO}$, $5 \mathrm{mM}$ BME, $100 \mathrm{mM} \mathrm{KCl}$, $19 \mathrm{mM} \mathrm{C}_{8} \mathrm{E}_{4}$ ) on a cover slip and equilibrated against $500 \mu \mathrm{L}$ of reservoir solution $(10 \%$ PEG6000, 10\% glycerol, 3\% t-BuOH, $100 \mathrm{mM}$ $\mathrm{LiSO}_{4}, 5 \mathrm{mM}$ BME) using hanging drop vapor diffusion method. It was sealed with immersion oil (Merck) and equilibrated at $19{ }^{\circ} \mathrm{C}$. Crystals were mounted in loops from mother liquid and flash frozen in liquid $\mathrm{N}_{2}$. The expression, purification, relipidation, crystallization and data collection of WT E2-AlF 4 was described in Andersen et al. ${ }^{22}$

\section{Structure determination}

Crystal diffraction data were indexed and integrated with $X D S^{37}$ and scaled with Aimless. ${ }^{38}$ The structure of $\mathrm{G}_{4} \mathrm{E}_{2}-\mathrm{BeF}_{3}^{-}$was determined by molecular replacement by Phaser $^{39}$ using a hybrid search homology model consisting of LMCA1 WT E2-AIF ${ }_{4}^{-},{ }^{22}$ where the $\mathrm{N}$ domain of LMCA1 was based on a homology model of rabbit SERCA E2$\mathrm{BeF}_{3}^{-}$(PDB: 3b9b). ${ }^{15}$ Homology models were made using an online version of Modeller (www.salilab. org/modeller). ${ }^{40}$ A sequence alignment of LMCA1 and SERCA performed in MUSCLE ${ }^{41}$ was used as an input file together with the PDB structures of $3 \mathrm{~b} 9 \mathrm{~b}$ and $3 \mathrm{~b} 9 \mathrm{r}$ for SERCA stabilized with $\mathrm{BeF}_{3}^{-}$ and $\mathrm{AlF}_{4}^{-}$, respectively. The structure determination was challenging, since pseudo-symmetry was present (Figure S1). $\mathrm{G}_{4} \mathrm{E}^{2}-\mathrm{BeF}_{3}^{-}$seemed to display orthorhombic symmetry, since the $\beta$ angle was close to $90^{\circ}$ and the self-rotation function at kappa $180^{\circ}$ showed three symmetry axes parallel to the crystallographic axes. However, the processing statistics clearly indicated that orthorhombic symmetry was not correct. The space group is the monoclinic $\mathrm{P} 2_{1}$, which only exhibits single two-fold symmetry. The self-rotation function at kappa $180^{\circ}$ reveals the presence of rotational pseudosymmetry parallel to the crystallographic symmetry axis. A native Patterson function ${ }^{42}$ analysis revealed a peak with a $29 \%$ height relative to the origin, which indicates the presence of translational pseudosymmetry. The non-crystallographic symmetry (NCS) operators close to true crystallographic symmetry operators made molecular replacement difficult, but it was eventually achieved, revealing an unusual packing of eight molecules in the asymmetric unit (Figure S1(A)). NCS averaging was used during refinement and the structure was refined at $3.0 \mathrm{~A}$ maximum resolution. Refinement was made challenging by the fact that large fractions of the $N$ domain were highly disordered in four out of the eight copies in the asymmetric unit (Figure S5). In fact, the asymmetric unit consists of both an ordered layer with low B-factors and a disordered layer with high B-factors. The disordered layer contributes to the relatively high average B-factor and explains the slightly higher R-factors than would be expected for a $3.0 \AA$ structure. The higher resoIution of $\mathrm{G}_{4} \mathrm{E} 2-\mathrm{BeF}_{3}^{-}$provided a better start model for structure determination of the WT forms and improving refinement with lower resolution data sets.

WT E2-BeF- and E2-AIF- crystals both exhibit $\mathrm{P} 2{ }_{1} 2{ }_{1} 2$ symmetry, The structure of WT E2- $\mathrm{BeF}_{3}^{-}$ was solved by molecular replacement using $\mathrm{G}_{4}$

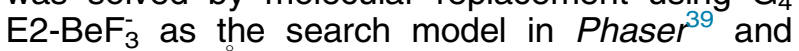
refined at $4.0 \AA$. For the WT E2-AIF- ${ }_{4}^{-}$structure, earlier obtained diffraction images ${ }^{22}$ were reprocessed in $X D S^{37}$ and scaled with Aimless. ${ }^{38}$ Molecular replacement in Phaser $^{39}$ solved the structure using WT E2- $\mathrm{BeF}_{3}^{-}$as a search model. Refinement of all of the structures was done in PHENIX ${ }^{43}$ and model building and analyses were performed in Coot. ${ }^{44}$

\section{CRediT authorship contribution statement}

Sara Basse Hansen: Investigation, Formal analysis, Validation, Writing - original draft, Writing - review \& editing, Visualization. Mateusz Dyla: Investigation, Resources, Formal analysis. Caroline Neumann: Formal analysis. Esben Meldgaard Hoegh Quistgaard: Investigation, Validation. Jacob Lauwring Andersen: Investigation, Resources. Magnus Kjaergaard: Methodology, Writing - original draft, Writing review \& editing, Supervision, Funding acquisition, Project administration. Poul Nissen: Conceptualization, Methodology, Writing - original draft, Writing - review \& editing, Supervision, Funding acquisition.

\section{Acknowledgements}

This work has been supported by grants to P.N. from the Lundbeck Foundation (DANDRITE-R2482016-2518) and the Independent Research Fund 
Denmark (FNU) (7014-00328B). S.B.H. was supported by a Ph.D. fellowship from the Boehringer Ingelheims Fond. The authors are grateful for early target identification by J. Preben Morth and the technical assistance of Anna Marie Nielsen and Tanja Klymchuk. We further thank the staff at the EMBL beamline P13 at the PETRA3 synchrotron in Hamburg for access and technical support.

\section{Declaration of Competing Interest}

The authors declare that they have no known competing financial interests or personal relationships that could have appeared to influence the work reported in this paper.

\section{Appendix A. Supplementary material}

Supplementary data to this article can be found online at https://doi.org/10.1016/j.jmb.2021. 167015.

Received 24 March 2021;

Accepted 22 April 2021; Available online 30 April 2021

$$
\begin{array}{r}
\text { Keywords: } \\
\text { Listeria; } \\
\mathrm{Ca}^{2+} \text {-ATPase LMCA1; } \\
\text { calcium; }
\end{array}
$$
membrane protein crystallography; P-type ATPase

$\dagger$ Present address: Hamlet Protein, Horsens, Denmark.

\section{References}

1. Dominguez, D.C., Guragain, M., Patrauchan, M., (2015). Calcium binding proteins and calcium signaling in prokaryotes. Cell Calcium, 57, 151-165. https://doi.org/ 10.1016/j.ceca.2014.12.006.

2. Dominguez, D.C., (2004). Calcium signalling in bacteria. Mol. Microbiol., 54, 291-297. https://doi.org/10.1111/ j.1365-2958.2004.04276.x.

3. Faxen, K., Andersen, J.L., Gourdon, P., Fedosova, N., Morth, J.P., Nissen, P., Møller, J.V., (2011). Characterization of a Listeria monocytogenes $\mathrm{Ca}^{2+}$ pump: a SERCA-type ATPase with only one $\mathrm{Ca}^{2+}$-binding site. $J$. Biol. Chem., 286, 1609-1617. https://doi.org/10.1074/jbc. M110.176784.

4. Rosch, J.W., Sublett, J., Gao, G., Wang, Y.D., Tuomanen, E.I., (2008). Calcium efflux is essential for bacterial survival in the eukaryotic host. Mol. Microbiol., 70, 435-444. https:// doi.org/10.1111/j.1365-2958.2008.06425.x.

5. Giotis, E.S., Muthaiyan, A., Blair, I.S., Wilkinson, B.J., McDowell, D.A., (2008). Genomic and proteomic analysis of the Alkali-Tolerance Response (AITR) in Listeria monocytogenes 10403S. BMC Microbiol., 8, 102. https:// doi.org/10.1186/1471-2180-8-102.
6. Dyla, M., Basse Hansen, S., Nissen, P., Kjaergaard, M., (2019). Structural dynamics of P-type ATPase ion pumps. Biochem. Soc. Trans., 47, 1247-1257. https://doi.org/ 10.1042/BST20190124.

7. Levy, D., Seigneuret, M., Bluzat, A., Rigaud, J.L., (1990). Evidence for proton countertransport by the sarcoplasmic reticulum $\mathrm{Ca}^{2+}$-ATPase during calcium transport in reconstituted proteoliposomes with low ionic permeability. J. Biol. Chem., 265, 19524-19534.

8. Cornelius, F., Møller, J.V., (1991). Electrogenic pump current of sarcoplasmic reticulum $\mathrm{Ca}^{2+}$-ATPase reconstituted at high lipid/protein ratio. FEBS Letters, 284, 46-50. https://doi.org/10.1016/0014-5793(91)80758-u.

9. Gong, D., Chi, X., Ren, K., Huang, G., Zhou, G., Yan, N., Lei, J., Zhou, Q., (2018). Structure of the human plasma membrane $\mathrm{Ca}^{2+}$-ATPase 1 in complex with its obligatory subunit neuroplastin. Nature Commun., 9, 3623. https://doi. org/10.1038/s41467-018-06075-7.

10. Dode, L., Andersen, J.P., Raeymaekers, L., Missiaen, L., Vilsen, B., Wuytack, F., (2005). Functional comparison between secretory pathway $\mathrm{Ca}^{2+} / \mathrm{Mn}^{2+}$-ATPase (SPCA) 1 and sarcoplasmic reticulum $\mathrm{Ca}^{2+}$-ATPase (SERCA) 1 isoforms by steady-state and transient kinetic analyses. $J$. Biol. Chem., 280, 39124-39134. https://doi.org/10.1074/ jbc.M506181200.

11. Dyla, M., Terry, D.S., Kjaergaard, M., Sørensen, T.L., Lauwring Andersen, J., Andersen, J.P., Rohde Knudsen, C., Altman, R.B., et al., (2017). Dynamics of P-type ATPase transport revealed by single-molecule FRET. Nature, 551, 346-351. https://doi.org/ 10.1038/nature24296.

12. Dyla, M., Kjaergaard, M., Poulsen, H., Nissen, P., (2019). Structure and Mechanism of P-Type ATPase lon Pumps. Annu. Rev. Biochem.,. https://doi.org/10.1146/annurevbiochem-010611-112801.

13. Winther, A.M., Bublitz, M., Karlsen, J.L., Møller, J.V., Hansen, J.B., Nissen, P., Buch-Pedersen, M.J., (2013). The sarcolipin-bound calcium pump stabilizes calcium sites exposed to the cytoplasm. Nature, 495, 265-269. https:// doi.org/10.1038/nature11900.

14. Sørensen, T.L., Møller, J.V., Nissen, P., (2004). Phosphoryl transfer and calcium ion occlusion in the calcium pump. Science, 304, 1672-1675. https://doi.org/ 10.1126/science.1099366.

15. Olesen, C., Picard, M., Winther, A.M., Gyrup, C., Morth, J. P., Oxvig, C., Møller, J.V., Nissen, P., (2007). The structural basis of calcium transport by the calcium pump. Nature, 450, 1036-1042. https://doi.org/ 10.1038/nature06418.

16. Ravishankar, H., Pedersen, M.N., Eklund, M., Sitsel, A., Li, C., Duelli, A., Levantino, M., Wulff, M., et al., (2020). Tracking $\mathrm{Ca}^{2+}$ ATPase intermediates in real time by $\mathrm{x}$-ray solution scattering. Sci. Adv., 6, eaaz0981. https://doi.org/ 10.1126/sciadv.aaz0981.

17. Gourdon, P., Andersen, J.L., Hein, K.L., Bublitz, M., Pedersen, B.P., Liu, X.Y., Yatime, L., Nyblom, M., et al., (2011). HiLiDe-systematic approach to membrane protein crystallization in lipid and detergent. Cryst. Growth Des., 11, 2098-2106. https://doi.org/10.1021/cg101360d.

18. Das, A., Rui, H., Nakamoto, R., Roux, B., (2017). Conformational transitions and alternating-access mechanism in the sarcoplasmic reticulum calcium pump. J. Mol. Biol., 429, 647-666. https://doi.org/10.1016/j. jmb.2017.01.007. 
19. Toyoshima, C., Nomura, H., Tsuda, T., (2004). Lumenal gating mechanism revealed in calcium pump crystal structures with phosphate analogues. Nature, 432, 361368. https://doi.org/10.1038/nature02981.

20. Sørensen, T.L., Clausen, J.D., Jensen, A.M., Vilsen, B., Møller, J.V., Andersen, J.P., Nissen, P., (2004). Localization of a $\mathrm{K}^{+}$-binding site involved in dephosphorylation of the sarcoplasmic reticulum $\mathrm{Ca}^{2+}$ ATPase. J. Biol. Chem., 279, 46355-46358. https://doi.org/ 10.1074/jbc.C400414200.

21. Olesen, C., Sørensen, T.L., Nielsen, R.C., Møller, J.V., Nissen, P., (2004). Dephosphorylation of the calcium pump coupled to counterion occlusion. Science, 306, 2251-2255. https://doi.org/10.1126/science.1106289.

22. Andersen, J.L., Gourdon, P., Møller, J.V., Morth, J.P., Nissen, P., (2011). Crystallization and preliminary structural analysis of the Listeria monocytogenes $\mathrm{Ca}^{2+}$ ATPase LMCA1. Acta Crystallogr., Sect. F: Struct. Biol. Cryst. Commun., 67, 718-722. https://doi.org/10.1107/ S174430911101548X.

23. Dyla, M., Andersen, J.L., Kjaergaard, M., Birkedal, V., Terry, D.S., Altman, R.B., Blanchard, S.C., Nissen, P., et al., (2016). Engineering a Prototypic P-type ATPase Listeria monocytogenes $\mathrm{Ca}^{2+}$-ATPase 1 for SingleMolecule FRET Studies. Bioconjug. Chem.,. https://doi. org/10.1021/acs.bioconjchem.6b00387.

24. Daiho, T., Danko, S., Yamasaki, K., Suzuki, H., (2010). Stable structural analog of $\mathrm{Ca}^{2+}$-ATPase ADP-insensitive phosphoenzyme with occluded $\mathrm{Ca} 2+$ formed by elongation of A-domain/M1'-linker and beryllium fluoride binding. J. Biol. Chem., 285, 24538-24547. https://doi.org/10.1074/ jbc.M110.144535.

25. Daiho, T., Suzuki, H., Yamasaki, K., Saino, T., Kanazawa, T., (1999). Mutations of Arg198 in sarcoplasmic reticulum $\mathrm{Ca}^{2+}$-ATPase cause inhibition of hydrolysis of the phosphoenzyme intermediate formed from inorganic phosphate. FEBS Letters, 444, 54-58.

26. Yamasaki, K., Daiho, T., Danko, S., Suzuki, H., (2015). Assembly of a Tyr122 hydrophobic cluster in sarcoplasmic reticulum $\mathrm{Ca}^{2+}$-ATPase synchronizes $\mathrm{Ca}^{2+}$ affinity reduction and release with phosphoenzyme isomerization. J. Biol. Chem., 290, 27868-27879. https:// doi.org/10.1074/jbc.M115.693770.

27. Toyoshima, C., Nakasako, M., Nomura, H., Ogawa, H., (2000). Crystal structure of the calcium pump of sarcoplasmic reticulum at 2.6 A resolution. Nature, 405, 647-655. https://doi.org/10.1038/35015017.

28. Karjalainen, E.L., Hauser, K., Barth, A., (2007). Proton paths in the sarcoplasmic reticulum $\mathrm{Ca}^{2+}$-ATPase. BBA, 1767, 1310-1318. https://doi.org/10.1016/j.bbabio.2007.07.010.

29. Bublitz, M., Musgaard, M., Poulsen, H., Thogersen, L., Olesen, C., Schiott, B., Morth, J.P., Møller, J.V., et al., (2013). Ion pathways in the sarcoplasmic reticulum $\mathrm{Ca}^{2+}$ ATPase. J. Biol. Chem., 288, 10759-10765. https://doi.org/ 10.1074/jbc.R112.436550.

30. Rui, H., Das, A., Nakamoto, R., Roux, B., (2018). Proton Countertransport and Coupled Gating in the Sarcoplasmic Reticulum Calcium Pump. J. Mol. Biol., 430, 5050-5065. https://doi.org/10.1016/j.jmb.2018.10.014.
31. Dolinsky, T.J., Nielsen, J.E., McCammon, J.A., Baker, N. A., (2004). PDB2PQR: an automated pipeline for the setup of Poisson-Boltzmann electrostatics calculations. Nucleic Acids Res., 32, W665-W667. https://doi.org/10.1093/nar/ gkh381.

32. Vangheluwe, P., Sepulveda, M.R., Missiaen, L., Raeymaekers, L., Wuytack, F., Vanoevelen, J., (2009). Intracellular $\mathrm{Ca}^{2+}$ - and $\mathrm{Mn}^{2+}$-transport ATPases. Chem. Rev., 109, 4733-4759. https://doi.org/10.1021/cr900013m.

33. Pedersen, B.P., Buch-Pedersen, M.J., Morth, J.P., Palmgren, M.G., Nissen, P., (2007). Crystal structure of the plasma membrane proton pump. Nature, 450, 11111114. https://doi.org/10.1038/nature06417.

34. Focht, D., Croll, T.I., Pedersen, B.P., Nissen, P., (2017). Improved model of proton pump crystal structure obtained by interactive molecular dynamics flexible fitting expands the mechanistic model for proton translocation in P-type ATPases. Front. Physiol., 8, 202. https://doi.org/10.3389/ fphys.2017.00202.

35. Musgaard, M., Thogersen, L., Schiott, B., (2011). Protonation states of important acidic residues in the central $\mathrm{Ca}^{2+}$ ion binding sites of the $\mathrm{Ca}^{2+}$-ATPase: a molecular modeling study. Biochemistry, 50, 1110911120. https://doi.org/10.1021/bi201164b.

36. Toyoshima, C., Norimatsu, Y., Iwasawa, S., Tsuda, T., Ogawa, H., (2007). How processing of aspartylphosphate is coupled to lumenal gating of the ion pathway in the calcium pump. Proc. Natl. Acad. Sci. USA, 104, 1983119836. https://doi.org/10.1073/pnas.0709978104.

37. Kabsch, W., (2010). Xds. Acta Crystallogr. D Biol. Crystallogr., 66, 125-132. https://doi.org/10.1107/ S0907444909047337.

38. Evans, P.R., Murshudov, G.N., (2013). How good are my data and what is the resolution?. Acta Crystallogr D, 69, 1204-1214. https://doi.org/10.1107/S0907444913000061.

39. McCoy, A.J., Grosse-Kunstleve, R.W., Adams, P.D., Winn, M.D., Storoni, L.C., Read, R.J., (2007). Phaser crystallographic software. J. Appl. Crystallogr., 40, 658674. https://doi.org/10.1107/S0021889807021206.

40. Sali, A., Blundell, T.L., (1993). Comparative protein modelling by satisfaction of spatial restraints. J. Mol. Biol., 234, 779-815. https://doi.org/10.1006/ jmbi.1993.1626.

41. Edgar, R.C., (2004). MUSCLE: multiple sequence alignment with high accuracy and high throughput. Nucleic Acids Res., 32, 1792-1797. https://doi.org/ 10.1093/nar/gkh340.

42. Patterson, A.L., (1935). A direct method for the determination of the components of interatomic distances in crystals. Z. Kristallogr., 90, 517-542.

43. Afonine, P.V., Grosse-Kunstleve, R.W., Adams, P.D., (2005). A robust bulk-solvent correction and anisotropic scaling procedure. Acta Crystallogr. D Biol. Crystallogr., 61, 850-855. https://doi.org/10.1107/ S0907444905007894.

44. Emsley, P., Cowtan, K., (2004). Coot: model-building tools for molecular graphics. Acta Crystallogr. D Biol. Crystallogr., 60, 2126-2132. https://doi.org/10.1107/ S0907444904019158. 